\title{
Serum vitamin-mineral profiles - association with breast cancer risk including dietary patterns and supplements use: a case-control study
}

\author{
Beata Krusinska $^{1}$, Lidia Wadolowska ${ }^{1}$, Malgorzata Anna Slowinska ${ }^{1}$, Maciej Biernacki ${ }^{2}$ and \\ Marek Drozdowski ${ }^{3}$ \\ ${ }^{1}$ Department of Human Nutrition, University of Warmia and Mazury, Olsztyn, Poland, \\ ${ }^{2}$ Department of Surgery, University of Warmia and Mazury, Olsztyn, Poland and \\ ${ }^{3}$ Department of Laboratory Medicine, University of Warmia and Mazury, Olsztyn, Poland
}

\begin{abstract}
Breast cancer is the most diagnosed cancer among women worldwide. Studies regarding diet and blood levels of vitamins and minerals in the breast cancer aetiology are limited and the results are inconclusive. We investigated the association between serum vitaminmineral profiles (V-MPs) and breast cancer (BC) risk with including dietary patterns (DPs) and use of supplements. This case-control study involved 420 women aged 40-79 years from north-eastern Poland, including 190 newly-diagnosed breast cancer cases. The serum concentrations of vitamins (folate, cobalamin, $25(\mathrm{OH})$ vitamin $\mathrm{D}$ ) and minerals (iron, calcium, magnesium) were marked in 129 post-menopausal women ( 82 controls, 47 cases) using an immune-analyser Cobas e411 and a Cobas Integra 400plus auto-analyser (Roche Diagnostics ${ }^{\circledR}$ ), respectively. A posteriori V-MPs were derived with a Principal Component Analysis (PCA). Three PCA-driven DPs: 'Non-Healthy', 'Prudent', and 'Margarine and Sweetened Dairy' were described previously. A logistic regression analysis was performed. The odds ratios (ORs) and $95 \%$ confidence interval $(95 \% \mathrm{CI})$ were calculated. Two serum V-MPs were identified. The 'Folate-Cobalamin-Vitamin D' profile was loaded heavily by the folate (factor loading 0.82), cobalamin (0.81) and vitamin D (0.49). The 'Iron-Calcium' profile was loaded heavily by the iron (0.81) and calcium (0.77). The 'Magnesium-vitamin D' profile was loaded heavily by the magnesium $(0.92)$ and vitamin $\mathrm{D}(0.39)$. The risk of $\mathrm{BC}$ was lower by $88 \%(\mathrm{OR}$ : $0.12 ; 95 \% \mathrm{Cl}$ : $0.02-$ $0.88 ; \mathrm{p}<0.05)$ in the upper tertile of the serum 'Iron-Calcium' profile compared to the bottom tertile. The risk of BC was lower by $67 \%(\mathrm{OR}: 0.33 ; 95 \% \mathrm{Cl}: 0.11-0.97 ; \mathrm{p}<0.05)$ at the level of serum $25(\mathrm{OH})$ vitamin $\mathrm{D} \geq 24.6 \mathrm{ng} / \mathrm{mL}$ and lower by $68 \%(\mathrm{OR}$ : $0.32 ; 95 \% \mathrm{Cl}: 0.11-0.91 ; \mathrm{p}<0.05)$ at the level of serum calcium $\geq 9.6 \mathrm{mg} / \mathrm{dL}$. There was the inverse association of the serum 'Magnesium-Vitamin D' profile with the risk of BC, which disappeared after adjustment for DPs. No significant association between $\mathrm{BC}$ risk and the serum 'Folate-Cobalamin-Vitamin D' profile and also folate, cobalamin, iron or magnesium considered separately was revealed. These findings highlight that the higher-adequate serum concentrations of both iron and calcium as well as close-to-adequate concentrations of calcium and vitamin D considered separately may protect against breast cancer among postmenopausal women, independently of dietary patterns and use of supplements. Therefore, women should be screened for blood concentrations of multiple vitamins and minerals in the breast cancer prevention.
\end{abstract}

\section{Conflict of Interest}

There is no conflict of interest. Project financially supported by Minister of Science and Higher Education in the range of the program entitled "Regional Initiative of Excellence" for the years 2019-2022, Project No. 010/RID/2018/19, amount of funding 12.000.000 PLN. 